\title{
Synaptic Noise and Other Sources of Randomness in Motoneuron Interspike Intervals
}

\author{
WIILIAM H. CALVIN AND CHARLES F. STEVENS
}

Department of Physiology and Biophysics, University of Washington

School of Medicine, Seattle, Washington

IT IS GENERALLY AGREED that most long-distance information transmission in the nervous system is accomplished by the propagation of all-or-none spikes along axons, and further that this information is carried in a single axon by the timing of spikes. When a neuron is discharging with a constant mean frequency, information is probably contained in the length of interspike intervals which, however, are notoriously variable. Much recent attention has been focused on the statistics of this variability. In part, this interest has reflected a concern for the accuracy of nervous system function (interval variability, from this point of view, represents noise in the signal being transmitted (8)), but perhaps a larger body of the literature (19) has been concerned with using the theory of stochastic processes to gain insight into the processes generating the spike trains.

To work backward from the statistics of interspike-interval variability to the mechanisms generating spike trains, however, involves making inferences about two separate mechanisms. Properties must be proposed for 1) the synaptic input of the neuron, and 2) the mechanism which converts synaptic input into spike trains. Variability in the interspike interval thus could be due to fluctuations in 1) the synaptic input, and 2) the mechanism converting synaptic currents into spike trains. Although Junge and Moore (15) were able to establish that intraccllularly observed fluctuations were consistent with the observed intcrval variability, they were forced to pool data from many Aplysia neurons whose firing frequencies were drifting. Thus none of the models in the literature has utilized the measured properties of either the steady-state synaptic input or of the spike-generating mechanism of the very neuron whose interval variability they wished to predict.

Received for publication November 6, 1967.
The purpose of the present work is to observe both the synaptic input and the conversion processes intracellularly and to evaluate their relative contributions to the interspikeinterval variability in a given neuron. Measurements of synaptic noise and of the observed properties of the spike generation process will be used to predict the interspikeinterval variability in the samc neuron; predicted and actual interval variability statistics will then be compared without the use of "free" parameters.

Because the experiments to be described require long duration and unusually stable intracellular recordings, we have concentrated our efforts on cat spinal motoneurons. When a steady current is injected into the spikegenerating region of these cells, either through the recording microelectrode or by prolonged synaptic action, they exhibit a type of repetitive discharge typical of many tonically firing receptors and central neurons (see Fig. 4): following a spike, the membrane repolarizes and then undergoes a linearly increasing depolarization that continues until the firing level is reached; at the firing level, a new spike is generated and the entire process is repeated. In this way a constant current gives rise to a repetitive spike discharge which can have a constant average frequency for a long period of time.

Although motoneurons often discharge with a constant mean frequency, they invariably exhibit irregular fluctuations in the time between successive spikes (the interspike interval). The preceding description of repetitive firing in motoneurons suggests at once (22) two broad classes of sources for this variability: 1) random factors in the spike-generating mechanisms such as firing level fluctuations, "noise" in the processes generating the linearly increasing depolarization, and fluctuation in the repolarization level; and 2) noise 
current superimposed on the constant current causing repetitive activity, including synaptic noise and thermal noise arising across the membrane impedance. We shall conclude that, for at least one class of motoneurons, synaptic noise alone is the major source of variability in the interspike interval, the other combined sources making contributions that are undetectable by our methods.

A brief report of this work has appeared previously (4), and additional details are available elsewhere $(2,2 \mathrm{a})$.

\section{METHODS}

This report is based on data collected from lumbosacral motoneurons in cats, either decerebrated or anesthetized with pentobarbital $(36 \mathrm{mg} /$ $\mathrm{kg}$ ). The cats were suspended by hip pins and a spinous process clamp attached to the L2 vertebra, and a laminectomy was performed to expose the spinal cord from L5 to the sacrum. After reflecting the dura in a manner which served to rotate the cord, the exposed area was covered with warm mineral oil. A vertebral body clamp at L6, shallow artificial respiration after a bilateral pneumothorax, and gallamine triethiodide paralysis were used to increase stability of the cord; to further stabilize the preparation, all clamps and electrode holders were mounted on the frame of a heavy stereotaxic instrument in which the animal was suspended. The animal's body temperature was monitored and maintained by intermittent heating.

Penetrations were made lateral to the dorsal root entry zone in the L7 region of the cord, and impaled cells were identified as motoneurons by antidromic activation. Usually, all dorsal and ventral roots were intact. Electrodes were relatively low-resistance (2-8 megohms measured in tissue with $0.5 \times 10^{-9} \mathrm{~A}$ brief current pulses) glass micropipettes filled either with $2.9 \mathrm{M} \mathrm{KCl}$ or $2.5 \mathrm{~m}$ potassium acetate. Signals were led through standard intracellular recording equipment, including a negative capacity preamplifier, and a bridge circuit permitted intracellular stimulation through the recording microelectrode. Data were recorded on tape (FM system, frequency response flat from 0 to $5 \mathrm{kHz}$ ) for analysis at a later time; both a lowgain d-c channel and a higher gain a-c channel (with spikes off scale) were recorded.

Neuron penetrations that would be perfectly acceptable for many purposes were unsuitable for the present investigation because we required long stretches of unusually stable behavior. Thus, neurons with small or medium-sized action or resting potentials were generally rejected at once; healthy cells that, at the time of the experiment, appeared to have a constant (mean) interspike interval were subjected to further examination. Data recorded included samples of synaptic noise in the absence of spikes and specimens of repetitive firing with several mean intervals (obtained by injecting constant currents of various magnitudes). After the experiment a list of intervals from the neuron was compiled in the computer and these were further screened for stationarity. Most cells did not yield a segment of at least 500 to 1,000 consecutive intervals with stationary statistics and were thcrefore rejected for further analysis. Stationarity was checked by inspecting a graph of successive intervals as a function of time for a run of data, and also by computing the first and second moments of the interspike-interval distribution for consecutive blocks of about a 100 intervals to see that they did not vary significantly with time. Because of these stringent requirements, relatively few of the 100 neurons studied were suitable for detailed analysis; this report is based primarily on 8 segments of data from the 5 best and most stable penetrations. The cells included were studied intracellularly for periods ranging from about $1 \mathrm{hr}$ to $5.5 \mathrm{hr}$.

Because we were concerned in certain parts of these experiments with measurements of synaptic noise, it is important to assess the amount of noise contributed by the instrumentation. The major fraction of instrumentation noise would be expected to arise from the electrode and negative capacity prcamplifier, and from the tape recorder. Noise from the first of these sources was minimized by using electrodes with as low a resistance as practical, e.g., 5 megohms; in this way thermal noise arising across the electrode resistance was at a minimum, and, because an adequate frequency response of the system could be obtained without heavy capacity negation, a relatively small amount of noise was added with the negative-capacity circuit. A maximally favorable signal-to-noise ratio for the tapc recorder was obtained by recording as large voltage swings as the system would accept. With these precautions the standard deviation of the noise voltage recorded when the electrode was in an extracellular position was always less than $50 \mu \mathrm{v}$. Because no dramatic increase of electrode resistance occurred on penetrating a cell, as judged by changes in bridge unbalance, it can be assumed that the component contributed by instrumentation to the recorded synaptic noise had a standard deviation on the order of $50 \mu \mathrm{v}$.

A LING computer was employed for all data analysis. Interspike intervals were initially compilcd in the computer by counting the time between spikes in $200-\mu \mathrm{sec}$ units and storing these interspike intervals in memory. After statistically stationary segments of data were located, the computer sampled the voltage records in these segments, converted membrane potential into eightbit words every $200 \mu \mathrm{sec}$, and stored them on computer tape. Spikes were allowed to go off scale so that the maximum gain could be obtained for the subthreshold voltage swings. 
This digitized membrane potential record was analyzed under visual control by displaying the voltage as a function of time on the oscilloscope display of the LINC. This phase of the analysis was carried out by the experimenter in a manner analogous to reading film, except that all measurements involved adjusting the value of program parameters with the LINC potentiometer knobs. When parameter adjustments were satisfactory a key was struck on the keyboard and the parameter values were stored automatically in memory. The firing levels were measured in this way by the experimeter who used, as the firing level criterion, the point at which the individual $200-\mu \mathrm{sec}$ points suddenly began to appear as separate dots on the screen. Similarly, a straight line could be manually fitted to the linearly rising membrane potential. The variability about this straight line could be computed automatically and compared to the synaptic noise in the quiescent state.

These semiautomatic data analysis methods had the advantage of allowing the experimenter to constantly view the data on which the computer was operating. Since no numbers needed to be written down by hand the method was quite rapid, especially as compared to the programming effort required to define some of the phenomena of interest. When anomalous features were noted in the data, e.g., sudden base-line shifts, accidental shock artifacts, etc., a sense switch could be raised before storing the measurements. This served to mark that datum in a special way so that it could be excluded from subsequent statistical treatment (or analyzed separately) if desired.

Once these intracellular measurements were stored on computer tape, scatter plots or probability distributions could be made using any of the following parameters: firing level, interspike interval, line slope, or line vertical position.

Models for the interval variability process were developed from examination of intracellular records. To test the model for a given motoneuron, not only must its assumptions (constant firing level, for example) be checked against the neuron's behavior, but the interval statistics of the model must be compared with those of the motoneuron. Whereas mathematical methods using first passage time equations may be applied to this problem $(3,22)$, it is usually casicr to simulatc the model process on the computer. The artificial intervals generated by the model may then be compared to those of the motoneuron, using all of the same computer programs by which the motoneuron intervals are processed. These "working models" operated in real time: deterministic components of the model were generated by programming, while random components were obtained by sampling noise voltages via the computer analogue input lines every $200 \mu \mathrm{sec}$.

\section{RESULTS}

\section{Synaptic noise}

All of the motoneurons encountered exhibited the haphazard fluctuations in their membrane potential known as synaptic noise. The amplitude of this noise was usually on the order of $2 \mathrm{mv}$ peak to peak, but in some cells it reached a peak-to-peak amplitude of 8 mv. Not only did the amplitude of the noise differ from cell to cell, but so did its appearance: in many neurons it had a rather structureless appearance and could plausibly have come from an appropriately filtered physical noise generator. In other cells individual EPSPs and IPSPs could be easily identified, and in some cases a few PSPs seemed to be the dominant components.

Such membrane potential fluctuations could conceivably be of either postsynaptic origin (such as thermal noise) or of presynaptic origin (either spontaneous release of transmitter or release by impulse activity arriving at the presynaptic terminals). Hubbard, Stenhouse, and Eccles (14) have shown that synaptic noise in kitten motoneurons probably arises predominantly from impulse activity arriving in the presynaptic terminals, since membrane potential fluctuations are significantly reduced by tetrodotoxin. That our synaptic noise is also of impulse origin is difficult to establish without a similar experiment, but the following observations suggest that the membrane potential fluctuations that we observed also arise as the temporal and spatial summation of PSPs caused by impulse activity. First, increased synaptic input obtained by stretching a muscle produces an increase in the noise amplitude. Second, increased depth of anesthesia results in a decrease in the noise amplitude, e.g., a reduction in noise variance from $7.8 \mathrm{mv}^{2}$ to $2.3 \mathrm{mv}^{2}$ with a supplementary dose of Nembutal equal to one-half the anesthetic dose. This indicates that at least two-thirds of the noise was of synaptic origin, assuming that the primary effect of the barbiturates was on synaptic inputs (18) and not on other possible sources of noise.

If one assumes random arrivals of PSPs it is possible to calculate from shot noise theory (20) the variability in the membrane potential to be expected $(1,2)$. When the average rate of occurrence of PSPs is sufficiently high 
the characteristic shape of a PSP can be completely obscured (since another PSP generally occurs before it can decay). Thus, though one can often identify individual PSPs in the synaptic noise, even homogeneous-looking noise can be of synaptic origin. For example, the noise data in Fig. 1 are from synaptic noise with a homogenous appearance. Figure $1 \mathrm{~A}$ (open circles) shows the amplitude distribution; the superimposed smooth curve is gaussian. Sincc amplitude distributions contain no information about how fast the membrane potential varies, we also use an autocorrelogram to measure the temporal characteristics of the synaptic noise. From Fig. $1 B$ it can be seen that the autocorrelogram is approximately exponential with a time constant of 4 msec. The open circles in Fig. $1 B$ are the autocorrelogram of white noise filtered by a singlestage RG filter. Since gaussian amplitude distributions and exponential autocorrelo-
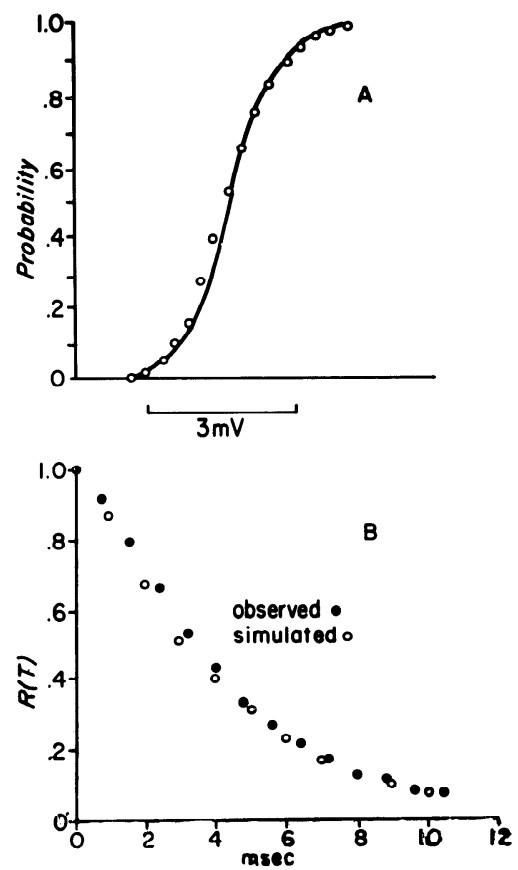

FIG. 1. Statistical structure of synaptic noise from motoneuron $M I V-I$. A: amplitude distribution histogram for a 2-scc sample (10,000 sample points) of synaptic noise using a class interval of $300 \mu \mathrm{v}$. The superimposed smooth curve is a normal distribution function with a SD of $0.8 \mathrm{mv}$. $B$ : autocorrelation function for a 30 -sec segment of synaptic noise sampled at $5 \mathrm{kHz}$ (filled circles). Open circles represent the autocorrelation function for filtered gaussian whitc noise used in the simulation of this cell's behavior.

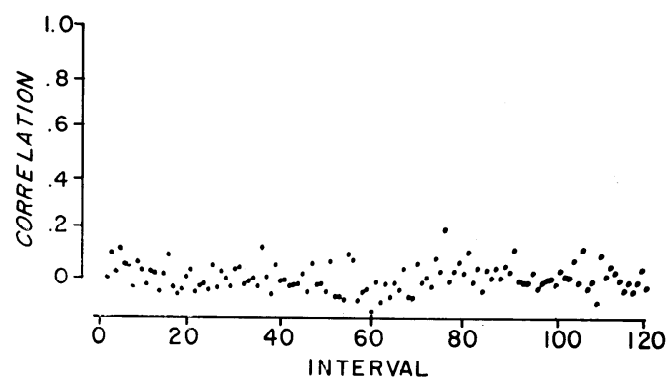

FIG. 2. Serial correlogram for a list of 1,536 consecutive intervals from cell $M I V-I$.

grams are predicted for rapidly arriving PSPs which sum linearly with each other, our homogeneous-appearing synaptic noise has a statistical structure consistent with temporal and spatial summation of small, rapidly occurring PSPs as the noise source.

\section{Interspike-interval variability}

All of the cells we have encountered have exhibited fairly pronounced interspike-interval variability, usually about $\pm 10 \%$ of the mean interval. As a general rule the variability has been of an approximately gaussian type, although we have found instances of multimodal interspike-interval histograms. We have never found a motoneuron which produced an exponential or longer-thanexponential (9) type histogram.

To completely specify a neuron's interspike-interval variability it would in general be necessary to specify the conditional probability of an interval as a function of all preceding intervals. One would not expect the entire past history of a neuron's behavior to be relevant, but the possibility that the preceding few intervals influence the duration of an interval certainly must be considered. Our task of describing motoneuron interspikeinterval variability was greatly simplified by the fact that, for the cells we have studied, each interval is independent of preceding intervals. This independence is revealed by serial autocorrelograms done for the lists of intervals studied; as the typical correlogram in Fig. 2 demonstrates, the autocorrelation is essentially zero for all but the self-correlations. For our motoneurons, then, the interspikeinterval histogram offers a complete probabilistic description of the cell's interspike-interval behavior. To account for the cell's variability it is nccessary only to account for the in- 


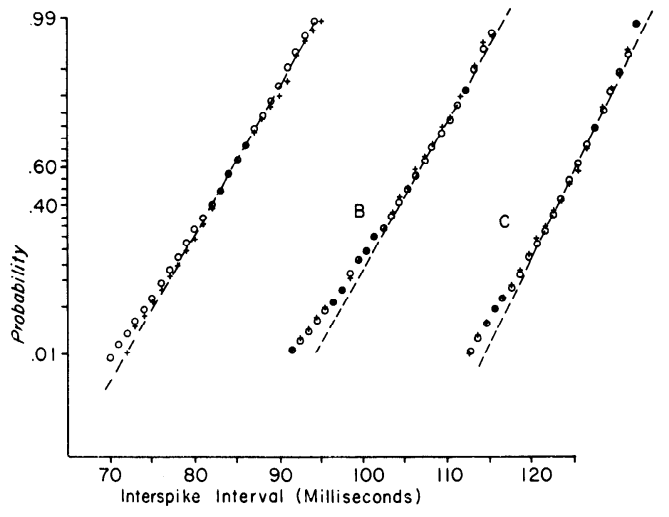

FIG. 3. Cumulated interspike-interval histograms for two motoneurons (crosses). One-millisecond class intervals were used, with sample sizes of 1,280 intervals for A and 590 intervals for B and C. Histograms $\mathrm{B}$ and $\mathrm{C}$ are from the same cell $(M I V-I)$ which provided data for Figs. 1, 2, and 4. Open circles are predictions made as described in the text; sample sizes were 3,072 for A and 1,536 for B and C. Vertical scale is such that a normal distribution appears as a straight line. A distribution is an integrated probability density; thus ordinary interspike-interval histograms (see Fig. 7) are first cumulated and then plotted on the probability paper to obtain the above graph.

terspike-interval histogram. Since we have dealt only with data meeting criteria for statistical stationarity, the variance of our interval histograms seems not to arise from drift or other similar causes; presumably the variance reflects random perturbations such as synaptic noise.

Because the presence of synaptic noise is such a conspicuous feature of all intracellular records, we expected from the outset that it would be an important contributor to the interspike-interval variability. To evaluate the magnitude of the contribution from this source, however, seems in general to be quite a difficult problem. The approach we have taken has been to develop an empirical description of the spike generation process (which we initially assume to bc deterministic), and then to predict the interspike-interval histogram which would result if random perturbations from synaptic noise were superimposed on this deterministic process. If the predicted and observed interspike-interval histograms agree satisfactorily, we conclude that synaptic noise provides an adequate explanation for the observed variability. If they do not agree, we try to discover why and to make our model of the process more realistic by including other possible influences. The method of procedure will become clear as it is applied to particular sets of data; a consideration of its implications will be postponed until the DIscussion section.

For convenience the motoneurons will be divided into two classes: the first class contains cclls whose firing level did not depend upon the duration of the interval, and the second class consists of cells with a systematic dependence of firing level on interval duration. A third section showing some of the exceptions to these two classes will illustrate more complicated behavior and indicate how its analysis may be approached.

\section{Class 1 motoneurons}

This section presents an analysis of three segments of data from two motoneurons. Interspike-interval histograms for the three segments of data are presented (crosses) in Fig. 3. All three histograms are approximately gaussian, but all are obviously skewed.

Because intervals from these cells are independent of each other, we need only to understand the interspike-interval histograms presented in Fig. 3 to account for the interspike-interval variability. Our first approach to the problem is to evaluate, by the procedure outlined earlier, the extent to which synaptic noise alone can produce the histograms; as indicated previously, this is accomplished by first developing a simple description for the spike generation process and then determining the effect the observed synaptic noise will have on the neuron's behavior.

In Fig. 4 superimposed traces of intracellular records from one of our neurons (the ccll that generated histograms B and C in Fig. 3) reveal that the terminal portion of the depolarization is plausibly fit by a straight line. Further, the firing level shows no very remarkable fluctuations although firing level determinations are also comparatively imprecise, as is clear from Fig. 4. A prcliminary model for spike generation, then, is a linear increase of depolarization to a constant firing level.

Synaptic noise would be expected to produce interspike-interval variability by causing variations in the time that the membrane potential first crossed the firing level (Fig. 4). To evaluate the interspike-interval variations expected from this mechanism we have carried out a computer simulation of the motoneuron's spike generation process in the fol- 
lowing way: first, the statistical structure of the synaptic noise was evaluated by determining the noise-amplitude histogram and its autocorrelation function (these are presented in Fig. 1). Second, the behavior of the cell was simulated on the computer by generating a linearly increasing "depolarization" (with the slope indicated by dashed line in Fig. 4) to which was added "synaptic noise" with the same statistical structure as the real noise. Each time the process crossed the "firing level" (indicated by horizontal dotted line in Fig. 4), the time since the last crossing was tallied, and the process restarted. In this way a sequence of artificial interspike intervals was generated. Finally, these predicted intervals were compiled into an interspike-interval histogram which was compared with the motoneuron's interval histogram (predicted points were plotted as open circles in Fig. 3). As is apparent from Fig. 3, the agreement between the observed and predicted histograms is excellent; according to the Kolmogorov-Smirnov goodness-of-fit test (5), the predicted and observed histograms are not statistically different at the .20 level. This means that one time in five, two histograms generated by the same random process would by chance differ more than the observed and predicted histograms in Fig. 3.

The simulation described in the preceding paragraph requires a number of parameters, such as the "slope" (average rate of depolari-
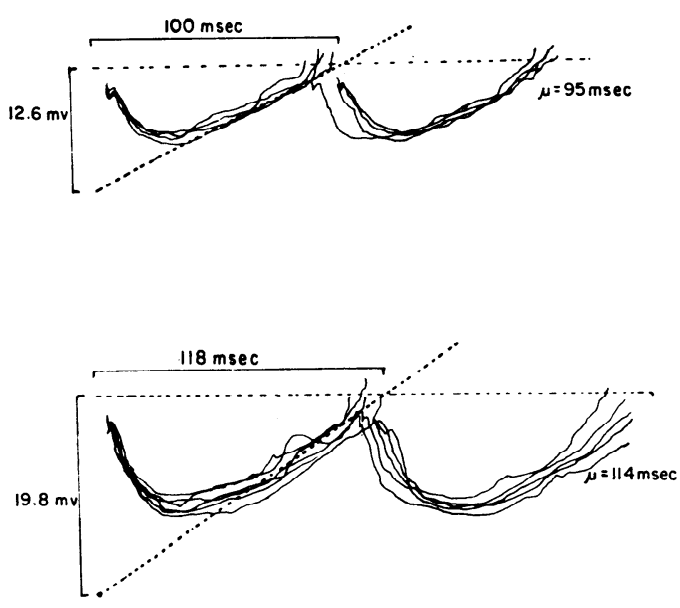

FIG. 4. Specimen tracings of intracellular recordings from motoneuron $M I V-I$, illustrating the behavior for two different mean firing frequencies. Dotted lines represent the deterministic model used in making the predictions presented in Fig. 3, B and C. zation), decay constant for the noise autocorrelogram, ctc., and it is important here to consider more fully how these parameters were obtained. In the case of the two cells represented in Fig. 3, the synaptic noise used in the simulation was noise from a commercial noise generator filtered to have the same autocorrelation function. Specifically, it was whitc gaussian noise passed through an RC filter which gave it the autocorrelation shown in the open circles in Fig. $1 B$. Thus, the characteristics of the noise added to the linearly increasing depolarization were determined completely by measurements on synaptic noise observed in the particular neuron under consideration. A preliminary estimate of the slope and firing level for the simulation was obtained by measurements from the rccordings of membrane potential like those illustrated in Fig. 4. After we obtained a first estimate of these parameters a trial simulation of the neuron's behavior was carried out, and the observed and predicted histograms were compared. Invariably, the predictions were close, but they were also significantly different by the Kolmogorov-Smirnov goodness-of-fit test. This difference results at least in part from the inevitable errors in estimating the slope and firing level. To discover if the synaptic noise is, within the errors of measuring constants (slope and firing level), adequate to accurately account for the observed histogram, these parameters were systematically varied within the range of the measurement error, further simulations were carried out, and the observed and predicted histograms were compared. The preliminary estimates of the two required constants were thus obtained from the intracellular recordings without reference to the observed interspike-interval histograms, and corrections to these constants were made within the range of measurements error of the original estimates; these corrections were less than approximately $10 \%$ of the preliminary estimate.

The discussion to this point has centered on the two histograms ( $B$ and $C$ ) in Fig. 3 which were obtained from the cell designated $M I V-I$. All of the procedures relating to histogram A from another cell are the same, with two exceptions. Because a sample of synaptic noise from the quiescent cell was not available the noise standard deviation was estimated from noise on the linearly increasing depolarizations involved in spike generation; 


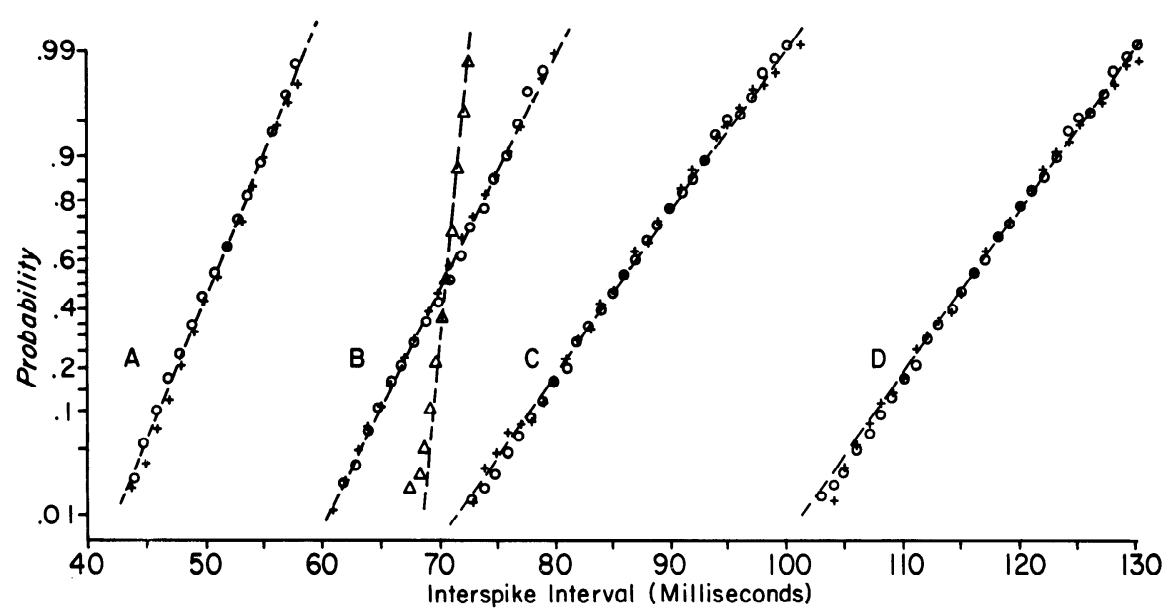

FIC. 5. Cumulated interspike-interval histograms from two motoneurons whose firing level increased systematically with time since the preceding spike (crosses). A and B are from one cell and C and D from another. Sample sizes are 737 for A, 768 for B, 512 for C, and 558 for D. For clarity of illustration, histogram B has been shifted $15 \mathrm{msec}$ to the right along the time axis. Open circles are predicted histograms computed as described in the text; in each case the predicted histograms were constructed from 768 "intervals" generated in simulating the motoneuron behavior. Open triangles in B are the predictions made by adopting a constant firing level (the neuron's average firing level) instead of linearly increasing ones as illustrated in Fig. 6.

this procedure has been validated with a number of cells from which synaptic noise samples are available and it has been found that the noise standard deviation in the quiescent cell is the same as that seen during activity (when activity is evoked by current injected through the electrode). Because it was not possible to perform autocorrelations on noise superimposed on the increasing depolarizations - the samples of noise obtained from the membrane potentials in the interspike interval are too short-we have used the same noise autocorrelation function obtained in the previous cell (Fig. 1B). Again, as is apparent from Fig. 3, the observed histogram is accurately predicted, within the range of error of estimating the required parameters from intracellular records. The predicted and observed histograms are not, according to the Kolmogorov-Smirnov test, different at the 0.10 level of significance. Therefore, for these motoneurons we would conclude that synaptic noise is sufficient to account for the observed interval variability.

\section{Class 2 motoneurons}

Figurc 5 (crosscs) presents four interspikeinterval histograms from two different motoneurons; in common with the class 1 cells the histograms are approximately gaussian. When firing level is plotted against length of interval
(Fig. 6) it becomes apparent that the firing level increases with interval length. An approximate description of spike generation for cells of this class, then, would consist of a linearly increasing depolarization to a firing level that is also increasing at a slower (but constant) rate. Plots of the type shown in Fig. 6 give no information about the behavior of the firing level for times shorter than those corresponding to the points located farthest to the left, except that the firing level must be sufficiently high so that the synaptic noise never crosses it at shorter times. As long as

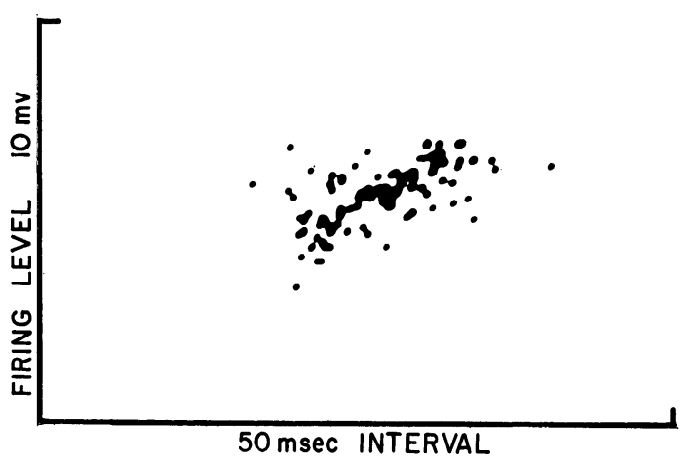

FIG. 6. Scatter plot of firing level vs. time since the preceding spike, for 200 of the intervals represented in Fig. 5A. A straight line fitted to this plot was used in making the predictions of Fig. 5A (open circles). Data from the cell of Fig. 5B yielded a similar scatter plot. 
the membrane potential has no chance of crossing the firing level it makes little difference what value it is assigned; for convenience we have made it constant to the left of the earliest points shown in Fig. 6, since that assumption is adequate to ensure that the observed membrane potential fluctuations were never large enough to cause crossing beforehand in simulations.

To estimate the effect produced by the observed synaptic noise in these cells, we used the same procedures as before, with two modifications. First, the firing level was made to increase at the rate estinnated from the plot shown in Fig. 6 instead of being constant as in the preceding case; the slope constant for the linearly increasing depolarization was estimated as before. Second, instead of using artificial synaptic noise made by filtering the output of a commercial noise generator, a tape-recorded segment of synaptic noise from the cell under consideration was sampled at $200-\mu \mathrm{sec}$ intervals and added to the computer-generated increasing depolarization. The result of the simulations of motoneurons' behavior is shown in Fig. 5: as before, the predicted and observed histograms appear to be in excellent agreement, an impression confirmed by the KolmogorovSmirnov goodness-of-fit test (.20 level). Final corrections of constants within the range of measurement error produced optimal agreement of predicted and observed histograms, with corrections less than $10 \%$ of the original estimates. For class 2 neurons, then, synaptic noise is sufficient to account for the observed interspike-interval variability.

An interesting indication of the sensitivity of the procedures used here is provided by using a constant rather than increasing firing level when simulating the behavior of one of these motoneurons. That is, the simulation leading to the good agreement in Fig. 5 is now modified only by adopting the cell's average firing level, i.e., a flat firing level as in class 1, thus ignoring the fact that it increases systematically with time. The result of this modification is illustrated in B of Fig. 5 (open triangles), where it is apparent that the agreement between predicted and observed histograms is entirely unsatisfactory. During the approximately 20 -msec period of time covered by histogram B in Fig. 5, the firing level increased only about $3 \mathrm{mv}$. This means that the constant firing level was in error by at most
$1.5 \mathrm{mv}$ during the time the membrane potential fluctuations had a substantial chancc of crossing the firing level, and yet this error caused a very marked change in the histogram. Not only was the predicted variance less than one-half the motoneuron variance, but the histogram became negatively skewed. If one did not know that the firing level systcmatically increased with time, it would have been tempting to suggest that there was another source of interval variability besides synaptic noise (such as firing level fluctuations). This illustrates how the parameters of the deterministic part of the model affect interval variability, and how hazardous it can be to reason about unseen sources of variability without measuring the characteristics of the spike generation process. In these cells, then, the only source of random behavior is still the synaptic noise: both components of the spike generator, the linear depolarization and the firing level, are still deterministic.

\section{Exceptions}

For the neurons considered in the preceding sections the principal source of interspikeinterval variability appeared to be synaptic noise; it is our impression that this conclusion probably holds also for many neurons that we could not analyze because they yielded an insufficient number of intervals with stationary statistics. It is natural to inquire if, in some instances, a source other than synaptic noise is the dominant cause of interspike-interval variability. Unfortunately, this question is very difficult to answer; one can state definitely that a noise source causes interval variability only when it is possible to account for the variability by using the measured properties of that source. Failure to account for the variability may mean only that one is using too simple a deterministic model for the spike generation process. For example, we could conclude that synaptic noise was the dominant source for interval variability in class 2 cells only when we had taken the accommodation into account. Had we been unaware of the steadily increasing firing level and assumed it to be constant (as for the triangles in histogram B of Fig. 5), then it would have appeared that synaptic noise accounts for only a fraction of the interval variability. In this case, then, the apparcnt need for another source would have been the result of an inappropriate deterministic model for 
spike generation. In another case where synaptic noise appears not to account for the observed variability, it might either be that a second noise source exists or that we have failed to model the spike-generating mechanism with sufficient accuracy. For instance, firing level might depend on the final rate of approach, or on some other property of the precise path taken by the increasing depolarization with superimposed noise. It seems not at all impossible that, in some neurons, the noise should interact with the spike-generating mechanism in some more complicated way than simple summation, as in the cases we have considered.

In this section we shall consider two instances where the simple model used previously fails. In one case the interspikeinterval histogram is dominated by haphazard occurrence of large events which are, at least at the descriptive level, quite different from anything considered earlier. In the second example we have identified a source of noise, presumably in the spike-generating mechanism, by measuring its properties and then including those properties in the simulation.

The upper part of Fig. 7 presents an interspike-interval histogram from the first of the exceptions we shall consider; rather than the approximately gaussian form encountered in earlier instances it is multimodal, with a number of identifiable peaks. It seems clear at once that the type of model employed earlier cannot predict this complicated behavior. The lower part of Fig. 7 gives sections of intracellular recordings from the cell that generated the histogram appearing above. These segments are aligned in time with the histogram and use the preceding spike as their reference time. Examination of the records reveals depolarizing wavelets that tend to occur at certain preferred intcrvals of about 17 msec, and that the peaks in the interspikeinterval histogram line up with the preferred time of occurrence of the wavelets. This correlation suggests that the haphazardly occurring wavelets are sometimes sufficiently large to bring the membrane potential to the firing level, thus giving higher probabilitics for spikes at "harmonics" of $17 \mathrm{msec}$. Here, then, is an additional source of variability that is quite different from that considered earlier. One possibility for the $17 \mathrm{msec}$ preference is, of course, 60 cycles/sec artifact. We have examined this possibility but can only conclude that if 60 cycle entered our batterydriven current source, it could have done so only in subnanoampere quantities at least one order of magnitude below those currents which normally cause action potentials (this conclusion follows both from the lack of 60 cycle visually observable in the records, and from considerations involving the design of the bridge circuit). If this is 60 cycle, then we would be tempted to conclude that it causes some component of the threshold kinetics to

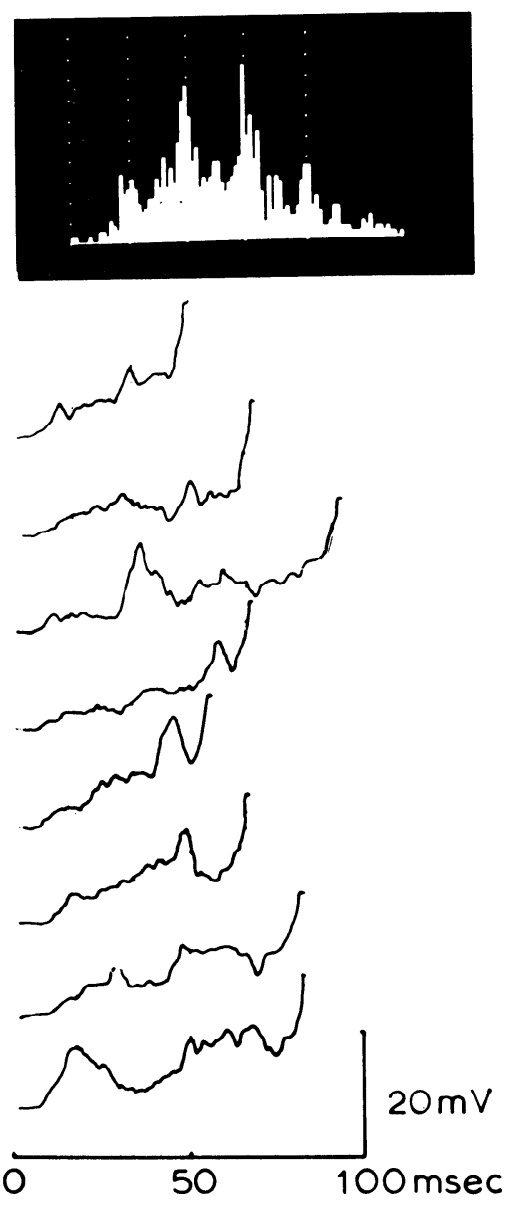

FIG. 7. Data from a motoneuron that produced a multimodal interspike-interval histogram. Upper part of figure shows the estimated probability density of a spike occurring at times following a spike at time zero (edge of histogram). A number of modes are present at multiples of 16-17 msec; thus, peaks occur at $34,50,66$, and $83 \mathrm{msec}$ following the spike at the origin. Tracings are from specimen membrane potential records that begin shortly following the previous spike. Wavelets in the interspike membrane potential are seen to occur frequently near these modal intervals, but sustained oscillations are absent. 
"lock in" to the 60-cycle frequency, even in such small quantities.

Another exceptional case is the motoneuron whose cumulated histogram appears in Fig. 8 (crosses). This histogram cannot be accounted for by the synaptic noise recorded from the cell, and the usual model of a linear increase of depolarization to the firing level (which in this case is constant): the predicted variance is much smaller than the observed variance and the observed and predicted skewnesses are in opposite directions (triangles in Fig. 8). More careful examination of the intracellular records reveals that the slope of the increasing dcpolarization changes, apparently randomly, from one interval to the next (i.e., the slope is steep, then shallow, etc. See Fig. 8). Here, then, is another source

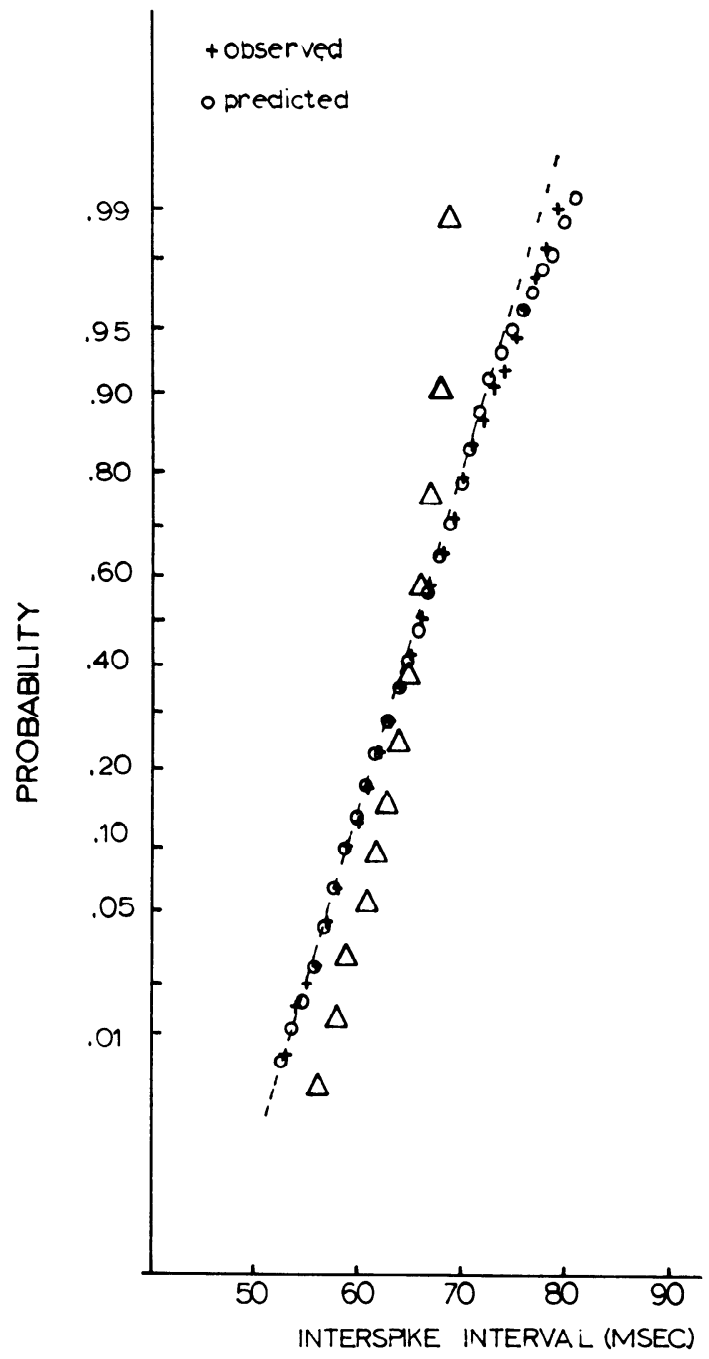

which can, along with synaptic noise observed in this cell, be evaluated as a contributor to the interspike-interval variability. Stimulation using 1) tape-recorded synaptic noise, 2) the firing level estimated from the intracellular recordings, and 3) a random sequence of slopes for the increasing depolarization (these were obtained by fitting straight lines to the spike generator depolarization in 35 successive intervals) yielded the cumulated histogram presented in Fig. 8 (open circles); the Kolmogorov-Smirnov goodness-of-fit test reveals the predicted and observed histograms are not significantly different at the .20 level. Thus the presence of synaptic noisc, together with random changes in depolarization slope from interval to interval, is adequate to account for the observed interspike-interval

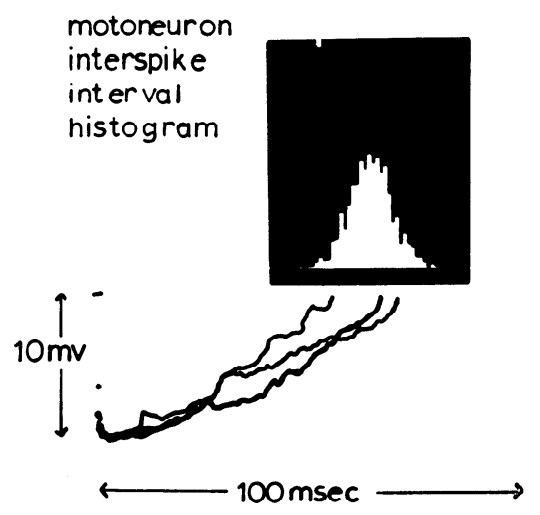

FIG. 8. Observed cumulative interspike-interval histogram from a motoneuron whose behavior could not be accounted for solely on the basis of synaptic noise (crosses). A noncumulated interspike-interval histogram is shown at right above specimen interspike membranc potential tracings. Tracings are aligned on the preceding spikes, and reveal that the terminal rate of approach to the firing level appears to vary from interval to interval. If this variation in depolarization rate was neglected in making predictions, too small a variance was obtained (open triangles), but if this slope variability was included in the model, the predicted histogram (open circles) is in adequate agreement with the observed behavior. The observed histogram and the predicted histogram shown with open triangles were constructed from 768 consecutive intervals, while the open-circle predicted histogram used 8,960 intervals. 
variability. In this case synaptic noise alone is insufficient, and a second source of variability has apparently been discovered. Without carrying out a detailed analysis of the sort described here it is impossible to state whether slope changes are important contributors to a given cell's variability; it is our impression that slope changes are less common sources of variability than is synaptic noise.

\section{DISGUSSION}

On the basis of evidence presented in the preceding sections we may conclude that synaptic noise, together with a descriptive model of the spike-generation process, can account quantitatively for the observed interval variability in certain motoneurons. It seems unlikely that these results are fortuitous because the predicted interspike-interval histograms had a variety of means and variances, and showed various degrees of skewness. Were synaptic noise not really related to the interval variability, one would not expect the correct shape of the histogram to be predicted in all cases studied. Thus we may suppose that, within the limits imposed by the experimental error, all of the variability arose from synaptic noise; further, the simplified model of spike generation is, again with the uncertainty of our measurement error, accurate for the situations in which we have employed it. We must now consider the probablc rcliability of these conclusions, and examine certain implications of our results for nervous system function.

Over all, we estimate that the error in evaluating our constants is less than approximately $10 \%$ of the mean value of the constant in question. The rangc of plausiblc values for the firing level and depolarization slope can be evaluated by examining Fig. 4; it is clear, we believe, that a $10 \%$ departure (in either slope or firing level) from the model indicated by the dashed lines would be outside the range of uncertainty in fitting the model. In cells for which tape-recorded synaptic noise was used in the simulations, there were, in effect, only two constants which entered into the simulation: the first was the ratio of synaptic noise standard deviation to the total amplitude of the increasing depolarization, and the second was the slope of the depolarization. When artificial synaptic noise was employed it was necessary also to select a value of the decay constant of the autocor- relogram (see Fig. 1B). Since we estimated that the mcasurcment crror in both constants combined is less than about $10 \%$ (see Fig. 4), our conclusions are uncertain to the extent implied by this error.

Although synaptic noise is the dominant noise source we cannot conclude that other noise sources are absent, or that they would not be important in other experimental conditions. Though one customarily measures amplitude fluctuations with the standard deviation, it is the variances (standard deviation squared) of independent random processes that add. Thus a noise source other than synaptic noise could have quite a large amplitude (standard deviation) and still make a relatively minor contribution to the total noise standard deviation. For example, if the firing level fluctuated with a SD of $0.5 \mathrm{mv}$ in a cell with a synaptic noise SD of $1.0 \mathrm{mv}$, the standard deviation of the total noise (synaptic plus firing level) would be only $1.12 \mathrm{mv}$. That is, a noise source half as large as synaptic noise would add only $12 \%$ to the standard deviation of the summed process. The $10 \%$ uncertainty in constants, then, means that we would not have detected a noise source up to almost $50 \%$ as large as the synaptic noise. On the other hand, the presence of even comparatively large but undetected sources does not materially affect our conclusion that synaptic noise is the dominant source for the variability in the class of cells under consideration. Under deep anesthesia, where synaptic input is considerably reduced, other noise sources (such as firing level variability) might dominate.

If synaptic noise were indeed the only noisc source then it is possible that our model of the spike-generating mechanism could be in error to the extent of about $10 \%$. This means, for example, that if firing level depends on the way it is approached, the total variation in firing level for the range of approaches seen in our neurons must be less than about $10 \%$ of the average value of the firing level (more precisely, the difference between the firing level and the maximum "repolarization" level obtained by extrapolating the linearly increasing depolarization back to the time of the preceding spike). Alternatively, if the synaptic noise interacts with the rate of depolarization the change in slope must not contribute more than $10 \%$ to the interval variability expected from synaptic 
noise. Altogether, any alteration in the spike generation model we have used that does not cause more than about a $10 \%$ change would be undetectable by our methods. In all probability our accuracy is somewhat better than the $10 \%$ quoted above, and the error is spread over several sources; thus additional noise sources are probably present, the increasing dcpolarization does not in fact increase quite linearly, firing level does depend to some small degree on the previous course of the membrane potential, etc. Because all of these corrections must fit within the approximately $10 \%$ uncertainty we feel that our account of interval variability is essentially correct.

Because the physiological implications of synaptic noise depend in part on its origin we must briefly examine the question: is synaptic noise of synaptic origin? The possible noise sources in a quiescent neuron include asynchronous activation of synapses by arriving impulses (10), central analogues of spontaneously occurring miniature end-plate potentials (16), thermal noise across the membrane impedance (7), "shot" noise arising from ions moving through the membrane, and other noise arising from the membrane (6). As noted in the RESUlTs section, synaptic noise is increased by procedures that increase synaptic input to a cell and is decreascd by anesthetics and tetrodotoxin, which presumably decrease input activity; these observations lend support to the notion that synaptic noise is primarily of synaptic origin. Further support also comes from estimating the contributions from certain of the other potential sources: calculations of the thermal noise arising in a (lumped model) motoneuron with a capacity of $3 \times 10^{-9}$ farads reveals that it should be less than $5 \mu \mathrm{v}$ rms. Further, if one assumes a 1-megohm cell resistance, takes the current necessary to cause a 20-mv depolarization, and supposes that ions jump quickly through the membrane according to a Poisson process, it can be shown that the shot noise contributed by this source would be less than $1 \mu \mathrm{v}$ rms. Thus these sources can be eliminated as significant contributors to synaptic noise. Finally, since frog node membrane noise (6) has quite a different autocorrelation from synaptic noise, this too can be tentatively eliminated. The remaining sourccs of noise are then spontaneous miniature PSPs, and those caused by tonic bombardment of the cell. Presumably, tonic bombardment is the major factor, especially in light of the effect of anesthetics and tetrodotoxin, so that it can be said that most of the observed interval variability is caused by irregular voltage fluctuations arising from asynchronous arrival of nerve impulses at the neuron's synapses.

The conclusion that impulse bombardment is the major source of noise has several implications for nervous system function. First, it means that noise in the nervous system is the unavoidable consequence of integration of information from various (unsynchronized) inputs. As long as spatial and temporal summation of PSPs are to be used by a neuron for integrating information, noise is the inevitable. Thus, noise is not an imperfection in the machinery of the nervous system which could be eliminated or at least minimized by proper "design" of spike-generating membrane; rather, it is inherent in the integration process. Second, because noise arises in the temporal and spatial summation of PSPs from diverse inputs, it places a fundamental limitation on the accuracy of nervous system operation. Thus, in order for an increase in the average depolarization of a neuron (decreasing the mean interspike interval) to be reliably detected within some relatively brief pcriod, the increase must be large compared to the standard deviation of the noise. The presence of noise, then, places limits on the ability to rapidly detect changes in the input to a cell, and the noise in turn arises from the steady inpul upon which changes are superimposed. The implications that noise in the nervous system have for psychophysics have been explored in recent years by psychologists (see, e.g., 13); our results indicate that there is a noise (and thus maximum accuracy for nervous system function) inherent in the use of finite-sized PSPs for integration of information.

At the level of the input-output rclation of a single motoneuron the significance of membrane potential variability has not been established experimentally, although the overall proportionality between firing frequency and injected current strength has been recently examined by several workers $(11,12$, 17). Some effect of synaptic noise on the slope of such frequency-current curves can be expected merely from the general properties of the time course of the membrane potential during repetitive firing (2). Furthermore, 
synaptic noise amplitude can affect accommodation measured with ramps of current $(21,23)$; extrapolating to the repetitive firing situation, synaptic noise amplitude might thus affect either the threshold for repetitive firing or the shape of the frequency-current curve. In the present work we have been concerned with some of the consequences of membrane potential variability at a single firing frequency. The success of the present analysis gives additional information about the spike-generating mechanism during repetitive firing. Thus, in the interval between spikes, the depolarization increases approximately linearly and perturbations of several millivolts do not seem to markedly affect this process. Further, the motoneurons do exhibit a firing level which is either constant or, in other cases, increases linearly with time. Again, the firing level is not significantly affected by membrane potential perturbations caused by synaptic noise. Any detailed theory of repetitive firing, then, must account for the type of behavior described here.

\section{S UMM A R Y}

Cat lumbrosacral motoneurons were studied with intracellular microelectrodes in an attempt to identify the sources of interspikeinterval variability observed in these cells.

All neurons studied exhibited the haphazard membrane potential fluctuations termed synaptic noise. Further, all neurons exhibited marked fluctuations in the interspike interval. The interspike-interval histograms were approximately gaussian with a standard deviation approximately $5 \%$ of the mean interval, and successive intervals were uncorrelated.

In one class of cells the spike-generator mechanism appeared to be adequately described by a model consisting of a linearly increasing depolarization that generates a spike on reaching a constant firing level and is then reset. In a second class of cell the firing level increased approximately linearly with time since the last spike, and this feature had to be included in the model of spike generation.

If measured properties of synaptic noise are combined with the measured parameters of the descriptive model for spike generation it is possible to make predictions of the neuron's interspike-interval variability. Close agreement of observed and predicted histograms was obtained for both classes of cells. It may thus be concluded that the presence of synaptic noise is adequate to account for the observed variability, and that other sources of variability make only negligible contributions.

In two instances synaptic noise alone was not adequate to account for the observed interspike-interval variability, but further analysis revealed an additional source that would account for the variability. In one case a multimodal histogram appeared to arise from "local responses" that tended to occur at preferred times. In the other instance the rate of depolarization appeared to change from interval to interval; by including this additional noise source it was possible to account for the observed variability.

\section{ACKNOWLEDGMENTS}

We express our thanks to Dr. J. Walter Woodbury and Dr. Albert M. Gordon for making their LINC computer available to us.

This research was supported by Public Health Service Grant NB 05934, with computer facilities supportcd by NB 01752 .

\section{REFERENCES}

1. Adolph, A. R. Feedback in physiological systems: an application of feedback analysis and stochastic models to neurophysiology. Bull. Math. Biophys. 21: 195-216, 1959.

2. Calvin, W. H. Motoneuron Input-Output Mechanisms: Analysis by Intracellular Recording (Ph.D. dissertation). University of Washington, 1966.

2a. Calvin, W. H. Evaluating membrane potential and spike patterns by experimenter-controlled computer displays. Exptl. Neurol. In press.

3. Galvin, W. H. and Stevens, C. F. A Markov process model for neuron behavior in the interspike interval. Proc. 18th Ann. Conf. Eng. Med. Biol. 7: 118, 1965.

4. Calvin, W. H. and Stevens, C. F. Synaptic

noise as a source of variability in the interval between action potentials. Science 155: 842-844, 1967.

5. Darling, D. A. The Kolmogorov-Smirnov, Cramer-von Mises tests. Ann. Math. Statist. 28: 823-833, 1957.

6. Derksen, H. E. Axon membrane voltage fluctuations. Acta Physiol. Pharmacol. Neerl. 13: 373-466, 1965.

7. Fatt, P. AND Katz, B. Some observations on biological noise. Nature 166: 597-598, 1950.

8. FitzHugh, R. A statistical analyzer for optic nerve messages. J. Gen. Physiol. 41: 675-692, 1958.

9. Gerstein, G. L. and Mandelbrot, B. Random 
walk models for the spike activity of a single neuron. Biophys. J. 4: 41-68, 1964.

10. Granit, R., Kellerth, J.-O., and Williams, T. D. Intracellular aspects of stimulating motoneurones by muscle stretch. J. Physiol., London 174: 435-452, 1964.

11. Granit, R., Kernell, D., and Lamarre, Y. Algebraical summation in synaptic activation of motoneurones firing within the "primary range" to injected currents. J. Physiol., London 187: 379399, 1966.

12. Granit, R., Kernell, D., and Lamarre, Y. Synaptic stimulation superimposed on motoneurones firing in the "secondary range" to injected current. J. Physiol., London 187: 401-415, 1966.

13. Green, D. M. And Swets, J. A. Signal Detection Theory and Psychophysics. New York: Wiley, 1966.

14. Hubbard, J. I., Stenhouse, D., and Eccles, R. M. Origin of synaptic noise. Science 157: 330 331, 1967.

15. Junge, D. AND MoORe, P. Interspike-interval fluctuations in Aplysia pacemaker neurons. Biophys. J. 6: 411-434, 1966.

16. KATZ, B. AND Miledi, R. A study of spontaneous miniature potentials in spinal motoneurons. J. Physiol., London 168: 389-422, 1963.
17. Kernell, D. The adaptation and the relation between discharge frequency and current strength of cat lumbosacral motoneurones stimulated by long-lasting injected currents. Acta Physiol. Scand. 65: 65-73, 1965.

18. L $\varnothing$ yning, Y., Oshima, T., and Yokota, T. Site of action of thiamytal sodium on the monosynaptic spinal reflex pathway in cats. $J$. Neurophysiol. 27: 408-428, 1964.

19. Moore, G. P., Perkel, D. H., and Segundo, J. P. Statistical analysis and functional interpretation of neuronal spike train data. Ann. Rev. Physiol. 27: 493-522, 1966.

20. Rice, S. O. Mathematical analysis of random noise. In: Selected Papers on Noise and Stochastic Processes, edited by N. Wax. New York: Dover, 1954.

21. Sasaki, K. And Oka, H. Accommodation, local response and membrane potential in spinal motoneurons of the cat. Japan. J. Physiol. 13: 508-522, 1963.

22. Stevens, G. F. Letter to the editor. Biophys. 7 . 4: 417-419, 1964.

23. Yamashita, H. Factors which decide the excitability of a spinal motoneuron in the cat. Japan. J. Physiol., 16: 684-701, 1966. 